\title{
Short-Term Seismic Activity in Vrancea. Inter-Event Time Distributions
}

\author{
Bogdan Felix Apostol ${ }^{*}, 1$ and Liviu Cristian Cune $^{2}$ \\ (1) National Institute for Earth Physics, Călugăreni Street, 12 Magurele, Ilfov, Romania \\ (2) National Institute of Physics and Nuclear Engineering, Magurele, Ilfov, Romania
}

Article history: received October 3, 2019; accepted November 14, 2019

\begin{abstract}
Short-term seismic activity in Vrancea is analyzed by means of the inter-event magnitude distributions (distributions of the next seismic event following a conditional seismic event). This seismic activity in Vrancea is fitted by Omori-type time-power laws, with possible correlations over a range of 20-25 days, for magnitudes $M<4-5$. Such temporal patterns can be employed in seismic hazard and risk estimation, providing the data set is statistically significant.
\end{abstract}

Keywords: inter-event time distributions; next-earthquake time-magnitude distributions; Omori law.

\section{Introduction}

The general goal of the statistical analysis of short-term seismic activity, especially the inter-event time distributions (next-earthquake, inter-occurrence, waiting time distributions), is to reveal possible regular patterns of earthquake occurrence (see, for instance, Bak et al., 2002, Lindman et al., 2005, Corral, 2004, 2005, and, especially, de Arcangelis et al., 2016 and References therein). Needless to say, such information is of relevance for seismic hazard and risk estimation. Short-term earthquake forecasting models were put forward [Reasenberg and Jones, 1989, 1994; Ogata, 1988, 1998; Gerstenberger et al., 2005; Omi et al., 2016, de Arcangelis et al., 2018], where universal scaling laws [Bak et al., 2002, de Arcangelis et al., 2016] have been discussed, possibly with a limited validity [Lindman et al., 2005], clustering and time-decreasing sequences governed by Omori-type power laws, or stochastic point-processes [Ogata, 1988, 1998]. We report herein upon similar statistical distributions for short-term seismic activity in Vrancea, focusing especially on their temporal behavior over short times. A general statistical analysis of the seismic activity in Vrancea has been reported previously [Apostol, 2006a, 2019].

A geometric-growth model has been introduced for energy accumulation in the focal region by Apostol [2006a]. Making use of this model, time, energy and magnitude distributions of earthquakes have been derived, in particular the well-known magnitude distribution law $\beta \mathrm{e}^{-\beta M}$. These results have been applied to a set of 1999 earthquakes with magnitude $M \geq 3$, which occurred in Vrancea between 1974 and 2004. This statistical analysis provided the value $\beta=1.89$. The analysis has been updated recently to 3640 Vrancea earthquakes with $M \geq 3$, corresponding to the period 1981-2018 [Apostol, 2019]. 


\section{Bogdan Felix Apostol et al.}

\section{General considerations upon temporal distributions of earthquakes}

It is generally accepted that earthquakes may conveniently be divided into background earthquakes and earthquakes accompanying main seismic shocks, as aftershocks and foreshocks. The background earthquakes are characterized by a mean recurrence time, depending on their energy (magnitude). The mean recurrence time has been interpreted as a failure time in a brownian-motion model [Matthews et al., 2002]. Poisson-like random processes may also be employed for the temporal distributions of the background earthquakes, as for rare events. They exhibit the well-known Gutenberg-Richter magnitude distribution $\beta \mathrm{e}^{-\beta M}$, where $M$ denotes the magnitude, which is well documented for various types of seismic activities, periods and regions. A typical value $\beta=1.17$ is provided for parameter $\beta$ by an isotropic point-like seismic focus model [Apostol, 2006 a,b], though different values are also known, like, for instance, $\beta=1.89$ for Vrancea seismic region [Apostol, 2006a]. Similarly, data given by Bullen [1963], indicate $\beta=1.38$ for a large set of global earthquakes. Variations in these parameters were reported, depending on the magnitude threshold [Godano et al., 2014]. The value $\beta=1.89$ corresponds to a geometric-growth model of an approximate faulting geometry for Vrancea focal region [Apostol, 2006 a,b]. Also, tectonic-style variability for this parameter has been reported for various seismic regions [Wiemer and Wyss, 2002; Giulia and Wiemer, 2010].

Background earthquakes represent a distinct seismic activity over long times. When a main shock is present, the seismic activity over short time is dominated by accompanying seismic events. Their temporal distributions are power laws of Omori type [Omori, 1894; Utsu, 1961], like 1/t, where $t$ is time measured with respect to the main shock, both in the future, as for aftershocks, and in the past, as for foreshocks (usually with a cutoff time). It is assumed, on theoretical grounds, that the accompanying seismic activity is symmetric under time reversal with respect to the main shock [Papazachos, 1978; Kagan and Knopoff, 1978; Jones and Molnar, 1979], although, in practical realizations, the past and future wings of these distributions are often unbalanced [Kazemian et al., 2015]. The pair distributions used here for our next-earthquake analysis are symmetric by their definition. The assumption that the analysis of the foreshock activity may shed light upon the occurrence of the main shock is questionable [Sornette et al., 1992; Sornette and Sammis, 1995; Sornette 1998; Helmstetter and Sornette, 2002, 2003; Felzer et al., 2004; Lippiello et al., 2012a, 2017; Seif et al., 2018]. Omori laws may arise from a self-replication phenomenon underwent by an original, exponential generating distribution [Apostol, 2006c], the aftershocks and foreshocks being distributed by a Gutenberg-Richter law in magnitude, as suggested previously [Vere-Jones, 1969]. Generating exponential distributions for the accompanying seismic activity have also been used to analyze Bath's law [Bath, 1965; Console et al., 2003; Helmstetter et al., 2004].

If the accompanying seismic activity is viewed as a relaxation process [Apostol, $2006 \mathrm{a}, \mathrm{b}$ ], its time distribution can be written as

$$
\frac{d p}{d|t|} \sim e^{-\alpha|t|}
$$

where $t$ is the time measured with respect to the moment of the main shock and $\alpha$ is a positive-valued coefficient of relaxation. The distribution given by equation (1) is symmetric in the parameter $\alpha$ and the statistical variable $|t|$. Therefore, we may assume that the parameter $\alpha$ is also a statistical variable, and we may set $\alpha=\gamma r$, where gamma is a positive constant and $r$ is a statistical variable. This corresponds to an ETAS model [epidemic-type aftershock sequence, Ogata 1988, 1998; Zhuang et al., 2011] with a continuous branching range, governed by $r$. Therefore, we have a two-variable distribution $P$, which can be written as

$$
\frac{d^{2} P}{d r d|t|} \sim e^{-\gamma r|t|}
$$

whence, by integrating with respect to $r$, we get

$$
\frac{d p}{d|t|} \sim \int_{0}^{\infty} d r \cdot e^{-\gamma r|t|}=\frac{1}{\gamma|t|}
$$


which is Omori power law. This is another derivation of Omori law, which is equivalent with the derivation that makes use of the self-replication process described by Apostol [2006c].

Omori's law represents only a particular pattern in the short-term seismic activity accompanying a main seismic shock, the seismic activity over a short-time scale being more complex. The scarcity of data regarding the foreshocks (i.e. the poor statistics) and the difficulty of identifying the aftershocks may render Omori's law of little practical use. In general, it is still missing a practical, reliable method of disentangling the truly accompanying seismic activity from other, background seismic activity, which may have not a direct relationship with the main shock, especially for the long tail of Omori's law (declustering problem). In addition, the shorttime seismic activity may exhibit a more complex pattern, of a multiple-branch character, indicating a superposition of Omori-type distributions. The short-time seismic activity may be described by a set of independent statistical variables, like, for instance, magnitude, time, location, etc., in a stochastic point-process approach, correlations included. Such short-term earthquake forecast models have been introduced in the fundamental works of Ogata, 1988, 1998. A parameter-decoupling scheme was used in the short-term sequenceclustering forecast model proposed for California [Gerstenberger et al., 2005; Reasenberg and Jones, 1989, 1994; Omi et al., 2016; de Arcangelis et al., 2018], which introduced a probability $P(M, t, r) \sim e^{-\beta M} \cdot \frac{1}{t_{c}+t} \cdot \frac{1}{\left(r_{c}+r\right)^{2}}$ for earthquakes characterized by magnitude $M$, occurrence time $t$, and location $r$, and written own this probability like a product of Gutenberg-Richter magnitude distribution and Omori-type distributions, where $t_{c}$ and $r_{c}$ are time and spatial cutoff parameters, respectively (for an isotropic spatial distribution). Such a probability law is fitted to empirical data, and employed for short-term prediction of daily rates of earthquakes. For a particular earthquake sequence the evolution parameters may be updated in real time, and employed for the next-day prediction.

\section{Next-earthquake correlation functions}

In view of the complexity of the statistical behavior of the short-term seismic activity, more general approaches may be convenient. Such approaches should be of more practical relevance, without resorting too much to patterns established statistically on general sets of data. From the practical standpoint the most relevant question in short-term earthquake forecasting seems to be "what happens next?". The general approach described herein is focused on this main question. It is based on inter-event time distributions employed for statistical analysis of short-term seismic activity. Suppose that an earthquake occurs at time $t_{0}$ and the next one occurs at some time $t$ measured with respect to $t_{0}$. Then we may define a distribution $P(t)$ of these next earthquakes, and determine it from a set of relevant statistical data. Once determined, it may be used for estimating the time probability of occurrence of the next earthquake, based on the principle "what happened will happen again". Characteristic scale time or size, or correlation range could be identified from the statistical analysis of such functions, providing the statistical set of data is large enough, which may shed light on the statistical patterns of a seismic activity. The statistics is rather poor, in general, precisely for that range of characteristic parameters where the estimation of the seismic hazard and risk is most interesting, like, for instance, for high values of magnitude $M$. The inter-event time distribution approach is applied here to earthquakes occurring in Vrancea, in order to illustrate its predictive capabilities and limits.

Temporal correlations exhibited by inter-event time distribution functions are described by gamma distributions, which imply power laws multiplied by exponentials [Corral, 2004, 2005]. Magnitude correlations have been demonstrated by a more elaborate model of dynamic scaling [Lippiello et al., 2008, 2012b; see also Stallone and Marzocchi, 2019]. Also, deviations from gamma distributions are reported [Bottiglieri et al., 2010; Lippiello et al., 2012c]. In general, the correlations involved in the short-term seismic activity are similar with those pertaining to accompanying seismic activity, which makes reasonable an Omori-type law for the former distributions.

\section{Brief characterization of Vrancea earthquakes}

The seismic area that is analyzed here is Vrancea, one of the most active intracontinental seismic areas in Europe. The earthquakes occur here within a volume with lateral extent $\sim 30 \times 70 \mathrm{~km}^{2}$ in the depth range $60-180$ $\mathrm{km}$. The rate of the seismic moment release is about $1.2 \times 10^{12} \mathrm{Nm} / \mathrm{yr}$ [Apostol, $2006 \mathrm{a}$,b]. Vrancea seismic region 


\section{Bogdan Felix Apostol et al.}

exhibits mainly middle-depth earthquake ( $\sim 80 \mathrm{~km}$ to $\sim 200 \mathrm{~km})$, and occasionally crustal, or surface, small earthquakes. Strong earthquakes occur sometimes in Vrancea, as, for instance, $M=7.4$, March 4, 1977, depth 94 $\mathrm{km}$, or $M=7.1$, August 30,1986, depth $131 \mathrm{~km}$. Nine earthquakes with magnitude $M>7$ have been recorded in Vrancea in the past two centuries. Although several tectonic models, spanning a large range of geodynamic scenarios, have been advanced to explain the characteristics of this undercrustal seismicity, the nature and mechanisms for earthquake generation are still subjects of debate. The statistical analysis of the associated distribution functions may provide information on the time and size patterns of earthquake occurrence if the statistical data set is large enough. A set of 1999 earthquakes with magnitude $M \geq 3$, occurred in Vrancea between 1974 and 2004 has been analyzed by using magnitude distribution of the type $\beta \mathrm{e}^{-\beta M}$ and exceedance rate, with $\beta=1.89$ and a total seismicity rate $1 / t_{0}=10^{4.21}$ per year, on average [Apostol, 2006a]. The basic data set used for the statistical analysis reported here (magnitude step $\Delta M=0.1$ ) includes 3640 earthquakes with (moment) magnitude $M \geq 3$ recorded between 1981 and 2018 (38 years) [ROMPLUS Catalog, 2018]. The magnitude distribution of these Vrancea earthquakes has been analyzed by using the Gutenberg-Richter law [Apostol, 2019], with $\beta \cong 2.1$ and $1 / t_{0 \cong 10^{4.92}}$ [error $\cong 10 \%$, Apostol, 2019].

\section{Inter-event time distributions for Vrancea}

For the sake of simplicity we neglect here the geographical and depth distribution of the earthquakes in Vrancea, because, on one hand, these earthquakes do not exhibit a large variability with respect to location coordinates, and, on the other hand, their inclusion may reduce considerably the size of the data set. Therefore, the generic parameter used in the analysis of the inter-event time distributions is the magnitude $M$. Seismic activity of the intermediatedepth source of region Vrancea (Romania) is analyzed here in terms of inter-event time-magnitude distributions. The data considered are seismic events with moment magnitude $3.0 \leq M$ (3640 events), which occurred during about four decades (since 1981 to 2018, 38 years). For this time interval and magnitude threshold, the ROMPLUS Catalog has been used [ROMPLUS Catalog, 2018].
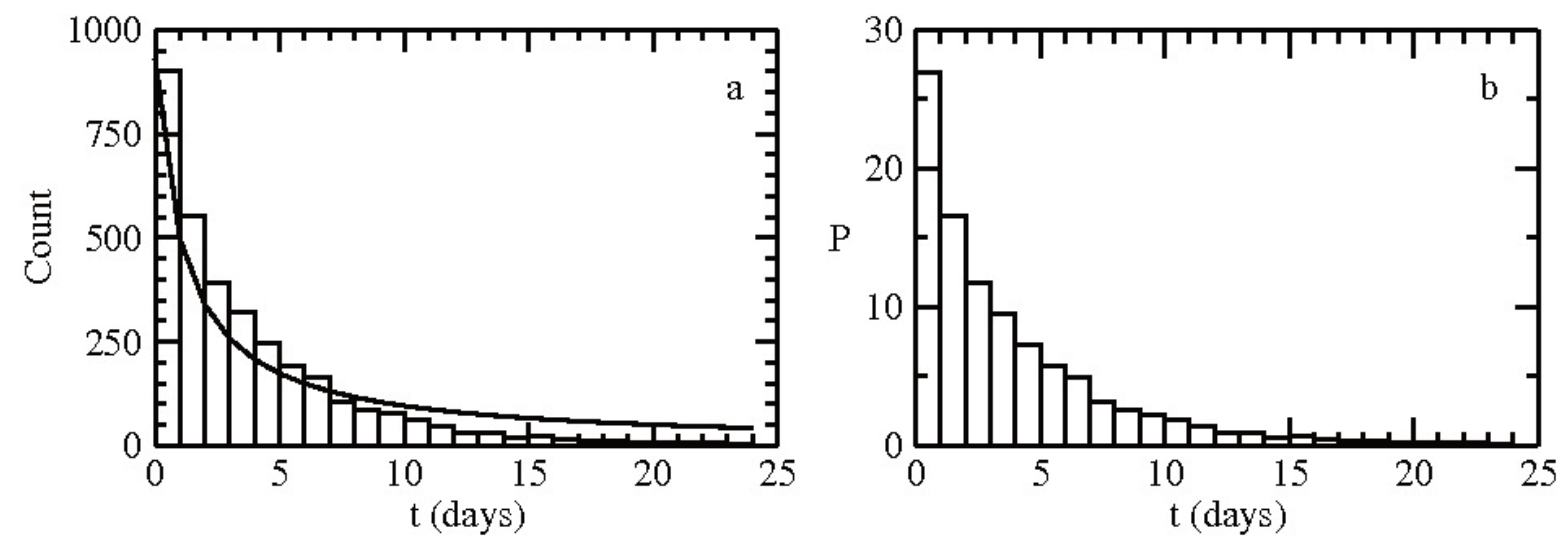

Figure 1. Inter-time distribution of seismic events in Vrancea 1981 - 2018 (3640 events, $M_{0}, M \geq 3$, panel $a$ ) and probabilities $P=P(t)$ (in \%, panel $b$ ). The fitting curve in panel $a$ is $1066.45 /(1.15+t)$ (determination coefficient 0.96$)$.

The seismic events with $M \geq 3$ are distributed in Figure 1 on the inter-event time (panel $a$ ); the corresponding probabilities $P(t)$ (in \%), calculated from the frequency of the events, are shown in Figure 1 panel $b$ (time is measured in days on the abscissa). We have taken all the earthquakes with magnitude $M \geq 3$ and have read from data how many days elapsed from each such earthquake to the next earthquake with magnitude $M \geq 3$ (a next-earthquake pair distribution). We can see that the rate of occurrence per day of the next earthquake follows a power-law time dependence similar to Omori's law, over a time window of about 25 days. The distribution is fitted with the law $a /(b+t)$, where $a=1066.45, b=1.15$ and $t$ denotes the time (coefficient of determination $R=0.96$; the coefficient of 
determination is defined as $R^{2}=1-\sum\left(d_{i}-f_{i}\right)^{2} / \sum\left(d_{i}-\bar{d}\right)^{2}$ where $d_{i}$ are the data, $\bar{d}$ is the mean value of the data and $f_{i}$ are fit values). The mean time for $P(t)$ is cca 5.89 days, and the variance is $\sigma=9.55$ days. We note the presence of the characteristic time $b$, which indicates a deviation from the gamma distribution, as pointed out recently [Lippiello et al., 2012c]. A fitting function $\sim \frac{1}{t^{\alpha}}, t>1, \alpha=0.25$, has been used previously by Apostol et al. [2008], for Vrancea, $M \geq 3$, time interval 1974-2004 (1999 earthquakes), with similar results. In a refined fit a decreasing exponential can be included, to account for the small values of the distribution tail. The data in Figure 1 have also been fitted with $A e^{-\gamma t} / t^{\alpha}$, where $A \simeq 683.3, \alpha \simeq 0.12, \gamma \simeq 0.22$. All these fits are of the same high quality. To check whether the results are biased by the aftershock sequences of the strongest seisms of the investigated time period (four earthquakes with moment magnitude $\geq 6.0$ ), two shorter time intervals have been considered: 1991-2018, avoiding the aftershock sequences of three events with $M>6.0$ (occurred on August 30, 1986, $M=7.1$, May 30, 1990, $M=6.9$, and May 31, 1990, $M=6.4$ ), and 2005-2018, when no earthquake larger than 5.6 moment magnitude occurred. The results show very similar forms of next-earthquake probabilities of occurrence, in all three cases ( $a=881.85$, $b=1.25, R=0.94$ for $1991-2018$ and $a=492.6, b=1.16, R=0.93$ for $2005-2018$ ).
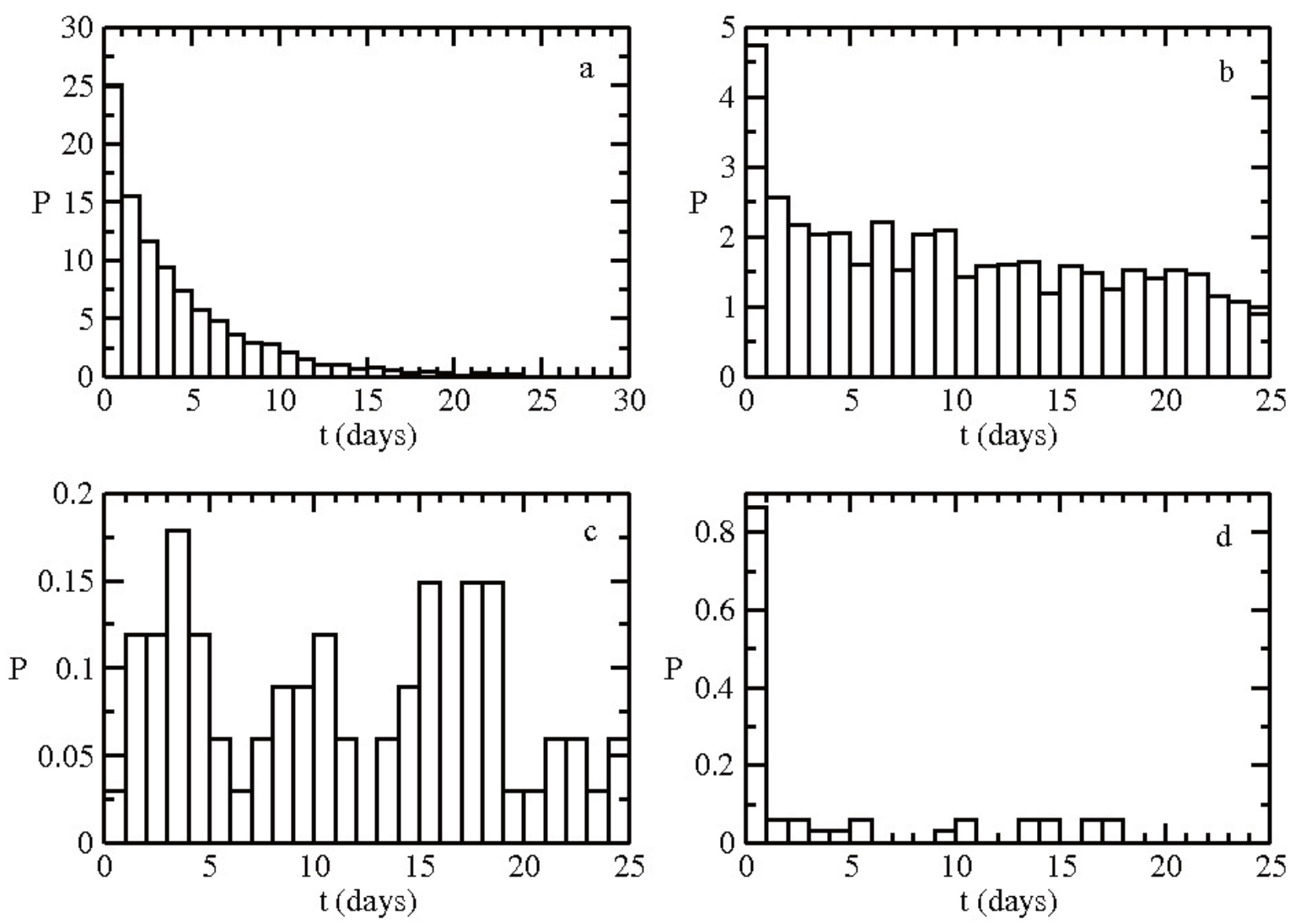

Figure 2. Inter-event time distributions $P=P(M, t)$ (in \%) for Vrancea 1981-2018 for $M_{0} \geq 3$ and $3 \leq M<4$ (panel $a$ ), $4 \leq$ $M<5$ (panel $b$ ), $5 \leq M<6$ (panel $c$ ) and $6 \leq M$ (panel $d$ ).

The probabilities $P(M, t)$ for $M_{0} \geq 3$ for Vrancea are shown in Figure 2 for $3 \leq M<4,4 \leq M<5,5 \leq M<6$, and $6 \leq M$ (panels $a, b, c$ and $d$, respectively). First, we note that the inter-event distributions $P(M, t)$ for Vrancea exhibit a characteristic decrease in time, with the highest probability of next-earthquake occurrence in the same day as the reference earthquake, at least for small magnitudes $(M<5)$. Then, we note the decreasing maximum values of these probabilities $\sim 22.7$ for $3 \leq M<4, \sim 2.75$ for $4 \leq M<5$, while the probability $P(M, t)$ vanishes practically for $M \geq 5$. This result agrees with the magnitude correlations discussed recently for conditional probability distributions [Lippiello et al., 2008, 2012b; Stallone and Marzocchi, 2019]. 
Bogdan Felix Apostol et al.
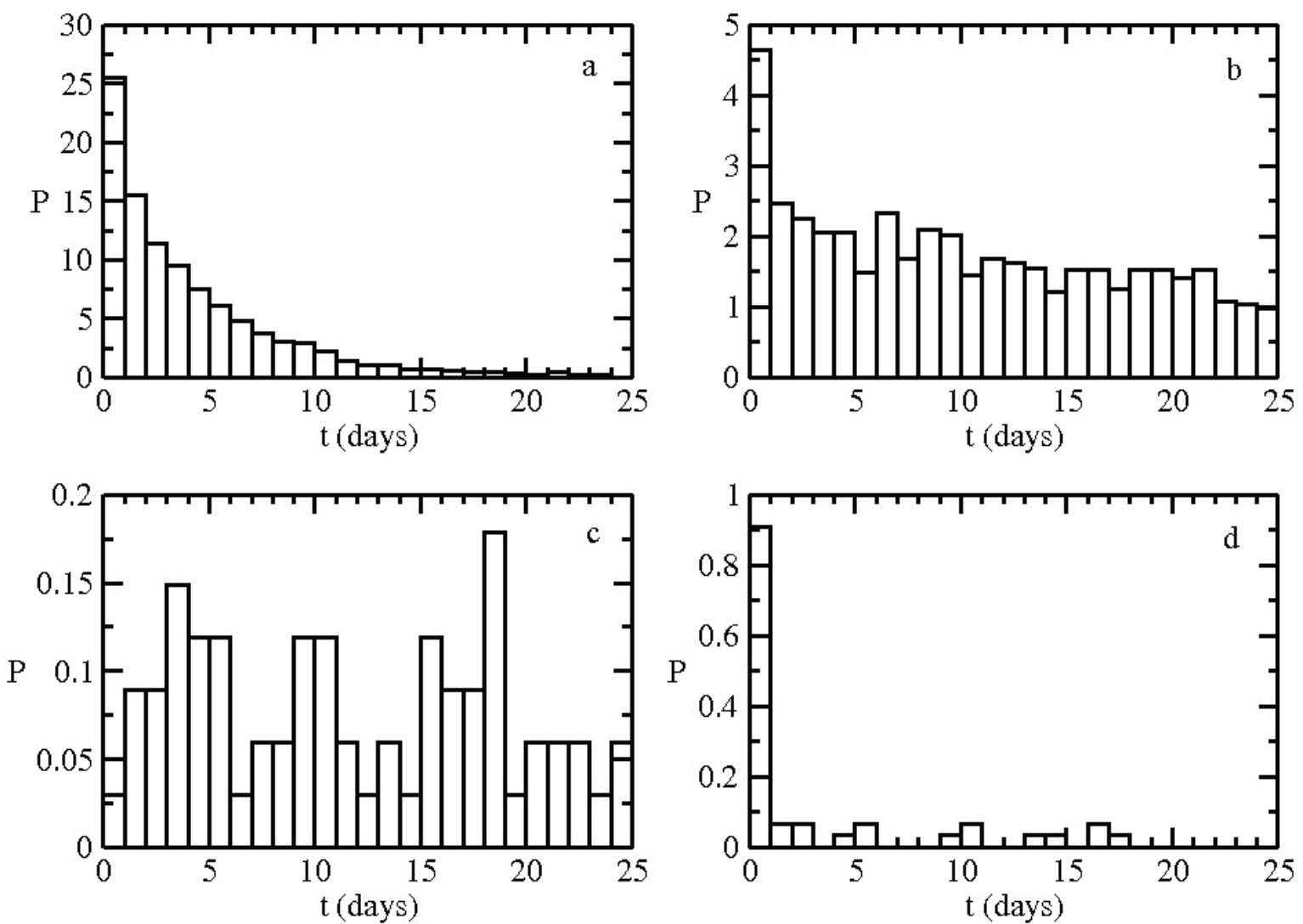

Figure 3. The inter-event time (conditional) probability $P=P\left(M, t \mid M_{0}\right.$ ) (in \%, Vrancea) for $3 \leq M_{0}<4$ and $3 \leq M<4$ (panel $a), 4 \leq M<5$ (panel $b$ ), $5 \leq M<6($ panel $c$ ) and $6 \leq M$ (panel $d$ ).
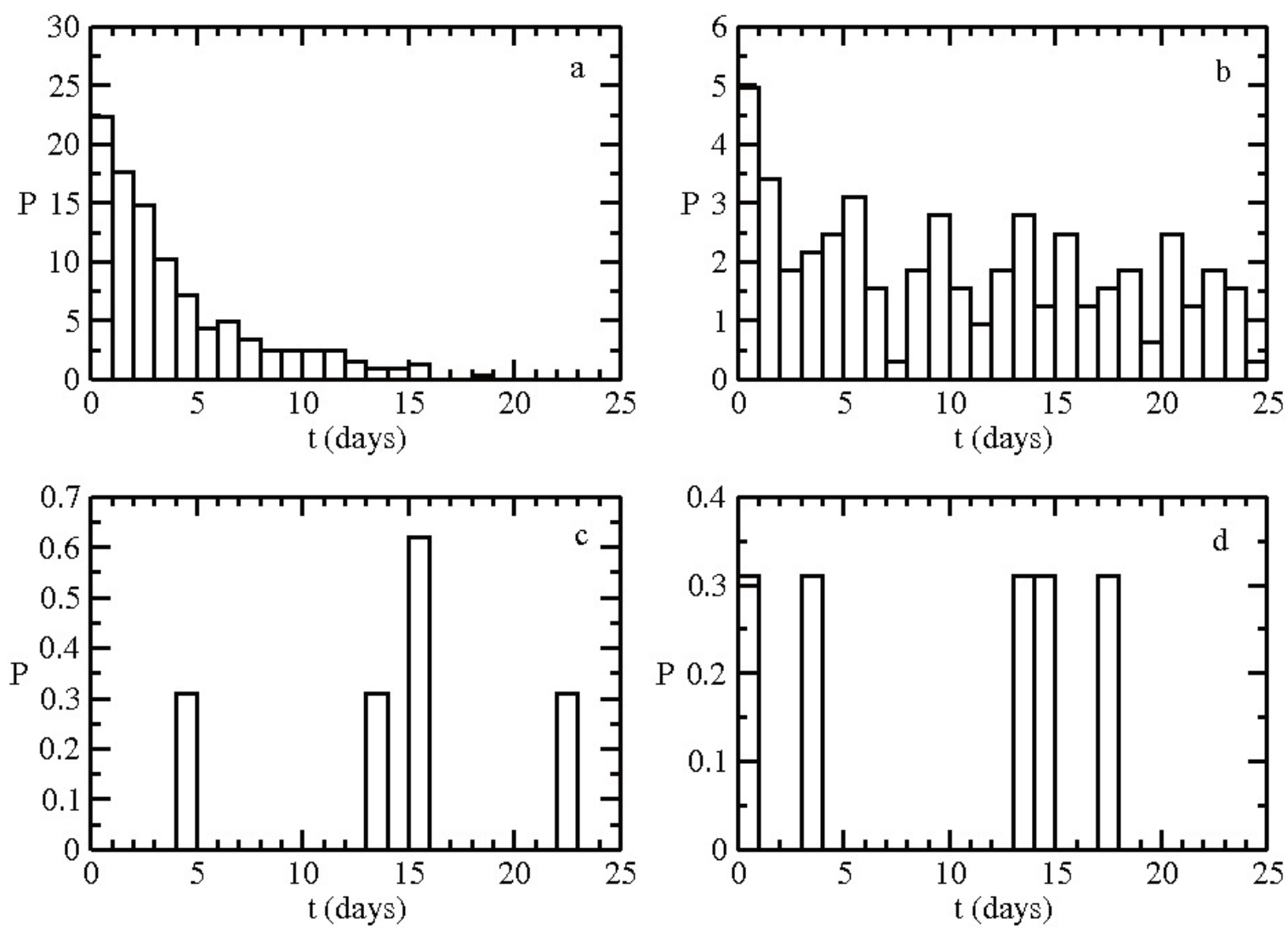

Figure 4. Same as in Fig. 3 for $4 \leq M_{0}<5$. 
Also, it is worth noting that $P(t)$ and $P(3 \leq M<4, t)$ are similar, obeying, Omori-type power laws, at least for short times, while the distributions become gradually irregular, exhibiting large fluctuations on increasing magnitude above $M=4-5$. The statistics becomes poor for higher magnitude $(M>5)$, as expected. A correlation time of 20-25 days can be estimated, after which the probabilities decrease appreciably (below 1), as well as a size correlation of cca $M=4-5$, above which the distributions acquire very small values, and are very irregular.

The conditional probabilities including the magnitude $M_{0}$ of the former earthquake $P\left(M, t \mid M_{0}\right)$ are shown in Figures 3-5 for $3 \leq M_{0}<4$ (Figure 3), $4 \leq M_{0}<5$ (Figure 4) and $5 \leq M_{0}<6$ (Figure 5). The first observation is that distributing the time-magnitude events with respect to the former earthquake magnitude $M_{0}$ does not change practically the characteristic time-decreasing behavior of the next-earthquake activity for small magnitudes.

It can be seen in Figure 2 (panel $a$ ) and Figure 3 that $P(M, t)$ and $P\left(M, t \mid 3 \leq M_{0}<4\right.$ ) are very similar, while

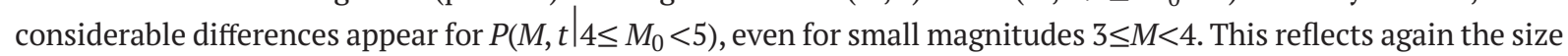
correlation $M=4-5$, and indicate large fluctuations for higher-magnitude distributions. Higher-order correlation functions (as well as higher-magnitude analysis, or enlarging the magnitude gap $\Delta M=1$ ) reduce considerably the statistical set, thus exhibiting a poor confidence.
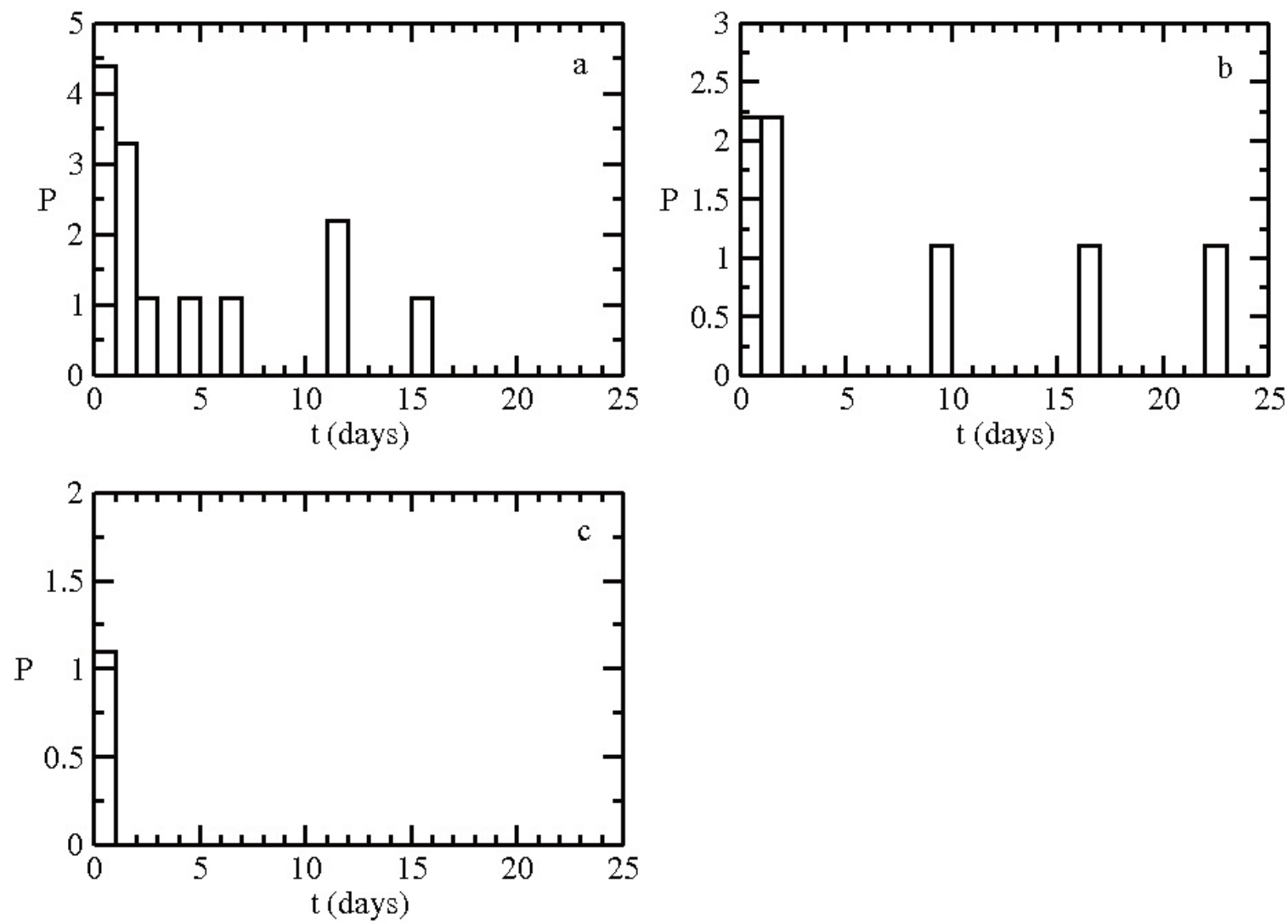

Figure 5. Same as in Figs. 3 and 4 for $5 \leq M_{0}<6$.

\section{Conclusions}

The inter-event time distributions are analyzed here for short-term seismic activity in Vrancea. The main distribution function is the time-magnitude probability $P\left(M, t \mid M_{0}\right)$ of the next earthquake of magnitude $M$ occurring at time t elapsed from the occurrence of the former with magnitude $M_{0}$ (inter-event time distribution). It may be viewed as a two-point correlation function, or a conditional probability. This distribution is obtained by analyzing 3640 earthquakes with (moment) magnitude $M \geq 3$ recorded in Vrancea over the last 38 years [since 1981 to 2018, ROMPLUS Catalog, 2018]. The results show a characteristic time-decreasing behavior of the 


\section{Bogdan Felix Apostol et al.}

occurrence of the next earthquake, at least for small magnitudes. Correlations are identified for lowermagnitude earthquakes ( $M, M_{0}<4-5$ ), extending roughly over 20-25 days. Statistics becomes poor for stronger earthquakes $(M>5)$, preventing any confident estimation. The results of the present report show that the interevent time distributions can be employed for estimating the short-term seismic hazard and risk for moderate earthquakes $(M<5)$ in Vrancea. For instance, from Figure 2 we read the probability 0.047 for occurring an earthquake with magnitude between 4 and 5 the same to the next day after one with magnitude $M \geq 3$ has occurred. In general, the approach can be useful for those seismic activities, periods and regions of interest, characterized by rich statistics (as, for instance, crustal, or surface, moderate earthquakes).

Data and sharing resources: http://www1.infp.ro/realtime-archive

Acknowledgements. The authors are indebted to dr. Luminita Angela Ardeleanu for enlightening discussions and to the anonymous referees who made valuable comments and suggestions. This work was partially carried out within the Program Nucleu 2019, funded by Romanian Ministry of Research and Innovation, project number PN19080102.

\section{References}

Apostol, B. F., (2006)a. A Model of Seismic Focus and Related Statistical Distributions of Earthquakes. Rom. Reps. Phys. 58, 583.

Apostol, B. F., (2006)b. A Model of Seismic Focus and Related Statistical Distributions of Earthquakes, Phys. Lett., A357, 462-466.

Apostol, B. F., (2006)c. Euler's Transform and a Generalized Omori's Law, Phys. Lett., A351, 3, 175-176.

Apostol, B. F., (2019). Statistical Seismology. NIEP internal report, Apoma MG, Magurele.

Apostol, B. F., L. C. Cune, and M. Apostol (2008). Scaling and universal power laws in time series of seismic events. Roum. J. Phys. 53, 3-4, 593-600.

Bak, P., K. Christensen, L. Danon, and T. Scanlon (2002). Unified Scaling Law for Earthquake, Phys. Rev. Lett., 88, 17, 178501.

Bath, M. (1965). Lateral Inhomogeneities in the Upper Mantle, Tectonophysics, 2, 6, 483-514.

Bottiglieri, M., L. de Arcangelis, C. Godano and E. Lippiello (2010). Multiple-Time Scaling and Universal Behaviour of the Earthquake Interevent Time Distribution. Phys. Rev Lett. 104, 158501.

Bullen, K. E., (1963). An Introduction to the Theory of Seismology, Cambridge, London.

Console, R., A. Lombardi, M. Murru, and D. Rhoades (2003). Bath's Law and the Self-Similarity of Earthquakes, J. Geophys. Res.: Solid Earth, 108, 2128, https://doi.org/10.1029/2001JB001651.

Corral, A. (2004). Long-Term Clustering, Scaling, and Universality in the Temporal Occurrence of Earthquakes, Phys. Rev. Lett. 92, (10), 108501.

Corral, A., (2005). Renormalization-Group Transformations and Correlations of Seismicity, Phys. Rev. Lett. 95 , (2), 028501.

de Arcangelis, L. C. Godano, C., J. R. Grasso, and E. Lippiello (2016). Statististical Physics Approach to Earthquake Occurrence and Forecasting, Phys. Reps., 628, 1-91.

de Arcangelis, L., C. Godano and E. Lippiello (2018). The Overlap of Aftershock Coda Waves and Short-Term Postseismic Forecasting, J. Geophys. Res.: Solid Earth, 123, 7, 5661-5674.

Felzer, K. L., R. E. Abercrombie and G. Ekstrom (2004). A common origin for aftershocks, foreshocks, and multiplets, Bull. Seism. Soc. Am., 94, 1, 88-98.

Gerstenberger, M. C., S. Wiemer, L. M. Jones and P. A. Reasenberg (2005). Real-Time Forecasts of Tomorrow's Earthquakes in California, Nature, 435, 328-331.

Gulia, L. and S. Wiemer (2010). The influence of tectonic regimes on the earthquake size distribution: A case study for Italy. Geophys. Res. Lett., 37, 10, L10305.

Godano, C., Lippiello, E. and de Arcangelis, L., (2014). Variability of the b Value in the Gutenberg-Richter 
Distribution, Geophys. J. Int. 199, 3, 1765-1771.

Helmstetter, A. and D. Sornette (2002). Sub-Critical and Super-Critical Regimes in Epidemic Models of Earthquake Aftershocks, J. Geophys. Res.: Solid Earth, 107, B10, 2237.

Helmstetter, A., S. Hergarten and D. Sornette (2004). Properties of Foreshocks and Aftershocks of the Nonconservative Self-Organized Critical Olami-Feder-Christensen Model, Phys. Rev., E70, 046120.

Helmstetter, A. and D. Sornette (2003). Importance of direct and indirect triggered seismicity in the ETAS model of seismicity, Geophys. Res. Lett., 30, 11, 1576.

Jones, L. M. and P. Molnar (1979). Some Characteristics of Foreshocks and their Possible Relationship to Earthquake Prediction and Premonitory Slip on Faults. J. Geophys. Res.: Solid Earth, 84, 3596-3608.

Kagan,Y. Y. and L. Knopoff (1978). Statistical Studies of the Occurrence of Shallow Earthquakes: the Two-Point Correlation Function, Geophys. J. R. Astron. Soc., 55, 67.

Kazemian, J., K. F. Tiampo, W. Klein and R. Dominguez (2015). Foreshocks and aftershocks in simple earthquake models, Phys. Rev Lett., 114, 088501.

Lindman, M., K. Jonsdottir, P. Roberts, B. Lund and R. Bodvarsson (2005). Earthquakes Descaled: on Waiting Time Distributions and Scaling Laws, Phys. Rev. Lett. 94, 10, 108501.

Lippiello, E., L. de Arcangelis and C. Godano (2008). Influence of Time and Space Correlations on Earthquake Magnitude, Phys. Rev. Lett., 100, 3, 038501.

Lippiello, E., W. Marzocchi, L. de Arcangelis, and C. Godano (2012)a. Spatial Organization of Foreshocks as a Tool to Forecast Large Earthquakes. Sci. Reps. 2, 846.

Lippiello, E., C. Godano, C. and L. de Arcangelis (2012)b. The Earthquake Magnitude is Influenced by Previous Seismicity, Geophys. Res. Lett., 39, 5, L05309.

Lippiello, E., A. Corral, M. Bottiglieri, C. Godano, C. and L. de Arcangelis (2012)c. Scaling Behaviour of the Intertime Earthquake Distribution: Influence of Large Shocks and Time Scales in the Omori Law, Phys. Rev. E86, 066119.

Lippiello, E., F. Giacco, W. Marzocchi, C. Godano and L. de Arcangelis (2017). Statistical Features of Foreshocks in Instrumental and ETAS Catalogs., Pure Appl. Geophys., 174, 1679-1697.

Matthews, M. V., W. L. Ellsworth and P. A. Reasenberg (2002). A Brownian model for recurrent earthquakes, Bull. Seism. Soc. Am., 92, 6, 2233-2250.

Ogata, Y., (1988). Statistical models for earthquakes occurrences and residual analysis for point processes, J. Amer. Statist. Assoc., 83, 401, 9-27.

Ogata, Y. (1998). Space-time point-process models for earthquakes occurrences, Ann. Inst. Statist. Math, 50, 379-402.

Omi, T., Y. Ogata, K. Shiomi, B. Enescu, K. Sawazaki and K. Aihara, (2016). Automatic Aftershock Forecasting: A Test Using Real-Time Seismicity Data in Japan, Bull. Seism. Soc. Am., 106, 6, 2450-2458.

Omori, F. (1894). Investigation of Aftershocks, J. Coll. Sci. Imper. Univ. Tokyo 7, 111-200.

Papazachos, B. C. (1978). Foreshocks and Earthquake Prediction, Tectonophysics 28, 4, 213-226.

Reasenberg, P. A. and L. M. Jones (1989). Earthquake Hazard after a Mainshock in California, Science 243, 4895, 1173-1176.

Reasenberg, P. A. and L. M., Jones (1994). Earthquake Aftershock: Update. Science, 265, 5176, 1251-1252.

ROMPLUS, (2018). National Institute for Earth Physics, Earthquake Catalog.

Seif, S., J. D. Zechar, A. Mignan, S. Nandan and S. Wiemer (2018). Foreshocks and their Potential Deviation from General Seismicity, Bull. Seism. Soc. Am., 109, 1, 1-18.

Sornette, D., C. Vanneste and L. Knopoff (1992). Statistical Model for Earthquake Foreshocks, Phys. Rev. A, 45, 8351.

Sornette, D. and K. G. Sammis (1995). Complex Critical Exponents from Renormalization Group Theory of Earthquakes: Implications for Earthquake Predictions, J. Physique, I 5, 607-619.

Sornette, D. (1998). Discrete-Scale Invariance and Complex Dimensions, Phys. Reps. 297, 239-270.

Stallone, A. and W. Marzocchi (2019). Empirical Evaluation of the Magnitude-Independence Assumption, Geophys. J. Int., 216, 2, 820-839.

Utsu, T. (1961). A Statistical Study on the Occurrence of Aftershocks, Geophys. Mag., 30, 521.

Vere-Jones, D. (1969). A Note on the Statistical Interpretation of Båth’s Law, Bull. Seismol. Soc. Amer., 59, 4, $1535-1541$. 


\section{Bogdan Felix Apostol et al.}

Wiemer, S. and M. Wyss (2002). Mapping spatial variability of the frequency-magnitude distribution of earthquakes, Adv. Geophys. 45, 259-302.

Zhuang, J., M. J. Werner, S. Hainzl, D. Harte and S. Zhou (2011). Basic Models of Seismicity: Spatiotemporal Models. Community Online Resource for Statistical Seismicity Analysis, doi:10.5078/corssa-07487583 (http://www.corssa.org).

"CORRESPONDING AUTHOR: Bogdan Felix APOSTOL, National Institute for Earth Physics, Călugăreni Street, 12 Magurele, Ilfov, Romania e-mail: apostol@infp.ro

๔) 2020 the Istituto Nazionale di Geofisica e Vulcanologia. All rights reserved 